\title{
REFLECTANCE SPECTRA FROM FREE-SWIMMING STICKLEBACKS (GASTEROSTEUS): SOCIAL CONTEXT AND EYE-JAW CONTRAST
}

\author{
by
}

\begin{abstract}
VICTOR N. RUSH ${ }^{1,2)}$, JEFFREY S. MCKINNON ${ }^{3,4)}$, MICHAEL A. ABNEY ${ }^{1,5)}$ and R. CRAIG SARGENT ${ }^{1,6)}$

$\left({ }^{1}\right.$ Biology Department and Center for Ecology, Evolution and Behavior, University of Kentucky, Lexington, KY 40506-0225, USA $;^{3}$ Department Biol. Sci., University of Wisconsin-Whitewater, Whitewater, WI 53190, USA)
\end{abstract}

(Acc. 7-VII-2003)

\begin{abstract}
Summary
The color patterns of many organisms change rapidly with social context but such dynamic signals have been little studied with current methods. In this study we applied objective spectrophotometry techniques to the color displays of unrestrained male threespine sticklebacks, to assess the influence of social context on coloration. Analyzing our data with a color space model based on stickleback visual physiology, we found that unrestrained males enhanced saturation of both their blue eye and red jaw color in response to the presence of a mature male or female conspecific. Divergence between the eye and the jaw lead to enhanced contrast, likely increasing conspicuousness. We found little relationship between measures of color saturation and condition, but the color of males in better condition varied more with social context. This study is the first to evaluate contrast between stickleback color pattern elements quantitatively and the first in which reflectance spectra were collected from freeswimming fish. The methods presented here could be used in future studies of sticklebacks and could potentially be adapted to other animals.
\end{abstract}

2) Present address: Tucker-Davis Technologies, 4637 NW 6th Street, Gainesville, FL 32605, USA.

4) Corresponding author's e-mail address: mckinnoj@uww.edu

5) Present address: US Fish \& Wildlife Service, 1339 20th Street, Vero Beach, FL 329603909, USA.

6) This research was supported by NSF 9728892 to Sargent, an NIH grant to Sargent and Rush and a University of Kentucky major equipment grant to Sargent. McKinnon was funded by an NSF Research Opportunity Award and by NSF 9981638. Charlie Baube and two anonymous reviewers provided helpful comments on the ms. 


\section{Introduction}

In fishes and other taxa, social stimuli may act through neural and hormonal mechanisms to trigger changes to pigment-bearing cells, and thus color patterns, within minutes or even seconds (Fuji \& Novales, 1969; Bagnara \& Hadley, 1973). Color patterns are clearly important in behavioral interactions and to fitness (Andersson, 1994), yet socially mediated color change has been surprisingly under-emphasized in recent behavioral studies. This is in part because it is difficult to analyze color patterns during social interactions with the techniques now recommended for visual signals (Endler, 1990; Bennett et al., 1994). In the work that has been done, most ethologists have either assessed the color of unrestrained animals subjectively or immobilized experimental subjects with restraints or anaesthetics. The limitations of the former method are well documented (Endler, 1990; see below also) but the immobilization necessary for collecting recommended spectral data may also be problematic, since it can cause potentially misleading changes to pigmentation patterns (McLennan \& McPhail, 1989a). In this study, we used a novel technique to collect spectral data from unrestrained, social sticklebacks. This made it possible for us to conduct analyses incorporating models based on stickleback visual physiology. This is the first evaluation of social influences on color expression, in a species with dynamic coloration, that uses current spectrophotometry methods and physiologically-based visual analyses.

The red-dominated nuptial coloration of the male threespine stickleback is both an example of a socially influenced display trait and one of the best known and best studied color signals in nature (reviewed by Rowland, 1994). Studies of stickleback coloration have built on classic ethological investigations (Tinbergen, 1951) to yield many important results in sexual selection (e.g. Milinski \& Bakker, 1990; Candolin, 1999a) and speciation (e.g. McPhail, 1969; Boughman, 2001). Most such studies have treated social context only incidentally, but work by McLennan \& McPhail (1989a, b, 1990) and Candolin (1999a, 2000) has shown that both red jaw intensity and blue eye coloration are adjusted in response to conspecifics, and that such adjustments may be adaptive. These important findings are limited, however, by either their reliance on human-based assessment of male coloration (directly with human eyes or using photographic systems that mimic human vision) or restraint of the subject males, or both.

It is important to analyze stickleback coloration in terms of the stickleback visual system because color vision in this species differs from that of 
humans in potentially significant ways. The key differences are the position of the long wavelength cone, which is shifted to a longer wavelength in sticklebacks; the increased separation between the middle and long wavelength cones (the close positioning of which, in humans, enhances detection of subtle shades of yellow and orange); and the addition of a fourth cone with peak sensitivity in the UV (Baube \& Loew, unpubl.). Clearly there are also substantial commonalities between human and stickleback color vision (reviewed by Frischknecht, 1993; McKinnon, 1995), so some methods involving technologies designed to mimic the human eye or evaluations by humans provide useful information (see also Bakker \& Mundwiler, 1994). Methods more closely allied to the stickleback visual system are preferable, however, and may lead to novel insights (Endler, 1990; Baube et al., 1995).

We used a color space model (Neumeyer, 1992) to examine how changes in the reflectance of the jaw and eye might be detected as differences in the proportional response of stickleback visual pigments. This simple model also allows us to test the possibility that contrast between elements of the stickleback color pattern is heightened during social interactions. Contrast between color pattern elements may be critical to color pattern conspicuousness (Endler, 1990; Brooks \& Endler, 2001), but previously this aspect of coloration had not been quantitatively evaluated in sticklebacks. Our approach also allows us to begin assessing the role that UV photoreceptors might play during social interactions (Cuthill et al., 1999; Losey et al., 1999; Smith et al., 2002).

Social interactions may influence not only coloration but also the relationships between coloration and condition or coloration and other traits of interest to conspecifics (Candolin, 2000). Consequently, we also utilize spectrophotometric data to re-evaluate widely cited but contentious earlier results (Milinski \& Bakker, 1990; Rowland, 1994) on the relationship between condition and color and to explore the influence of social context on the dynamics of condition-dependent signaling in sticklebacks (Candolin, 1999b).

\section{Methods}

Experimental subjects

Anadromous threespine sticklebacks were collected early in the spring breeding season from the Little Campbell River, Surrey, British Columbia (Hagen, 1967). They were transported by air to the Sargent laboratory in Lexington, Kentucky, after a brief stay in the McKinnon 
laboratory in Whitewater, Wisconsin. In the laboratory, all fish were maintained in cool spring conditions, approximately $14^{\circ} \mathrm{C}$ with a 16 light : 8 dark diurnal cycle (e.g. McKinnon, 1995) in 100 liter mixed sex holding tanks. They were fed twice daily on a mixture of frozen brine shrimp and red worms. Males to be used in experimental trials were measured for length then transferred individually to 76-liter tanks provided with nesting materials. They were encouraged to build nests and court through daily exposure to females of the same population (e.g. McKinnon, 1996), then allowed to spawn with a single female. At the conclusion of each experimental trial each male was weighed. Condition was calculated using the standard fisheries 'Fulton' index $\left(100,000^{*}\right.$ Weight $(\mathrm{g}) /$ Length $(\mathrm{mm})^{3}$ : Bolger \& Connolly, 1989) which, for our data set, correlated very strongly with other measures of condition $(r=0.89$ with residuals from regression of weight on length, 0.90 for residuals of log weight on log length).

\section{Stimulus presentation}

Stimulus presentation occurred three days after spawning. Males spend some time in parental activities by this point and their frequent nest visits enable collection of reflectance data (see below); but they continue courtship (Wootton, 1976; Kraak et al., 1999) and therefore retain most nuptial coloration. A stimulus consisted of a $500 \mathrm{ml} \mathrm{jar} \mathrm{that} \mathrm{contained} \mathrm{(1)} \mathrm{no} \mathrm{fish;} \mathrm{(2)} \mathrm{a}$ smaller male; or (3) a gravid female. To provide time for male coloration to stabilize, the male was allowed fifteen minutes after his first approach to the stimulus before collection of reflectance data. Each of the two fish stimuli was immediately preceded by a no-fish stimulus with a three-hour reacclimation period between the first fish stimulus and the second no-fish stimulus. Order of fish stimulus presentation (i.e. male or female first) was alternated among individuals and was found not to alter cone stimulation results for the no-fish stimulus (paired $t$-test, $p>0.4$, in all cases, without Bonferroni correction, $N=10$ ).

\section{Collection of reflectance data}

Four 50-watt Solux-daylight mimicking halogen lamps and a $20 \mathrm{~W}$ black flourescent light illuminated the test-tank, producing enough illumination for reliable reflectance measurements between $350 \mathrm{~nm}$ and $725 \mathrm{~nm}$. We used a Nikon UV lens on a macro tube to feed light via a fiber optic into an Oriel Instaspec IV ${ }^{\mathrm{TM}}$ CCD attached to a FICS ${ }^{\mathrm{TM}}$ (fixed image compact spectrograph). We were able to measure the reflectance of small color patches (approximately $2 \mathrm{~mm}$ diameter) on the focal male as he fanned eggs at the nest. Each reflectance measurement lasted 660 milliseconds (ms) and consisted of ten consecutive scans (66 ms each) of the same region, with a measurement accuracy of $3 \mathrm{~nm}$. Up to five such measurements were collected from each of (1) the jaw, specifically the red patch at the base of the mouth; (2) the eye, from the most posterior point forward. Light measurements from each body part were converted to reflectances through reference to analogous measurements from a Spectralon ${ }^{\mathrm{TM}}$ white standard placed in the same position and orientation in the tank as the male. Reflectance measurements were discarded if the animal moved during the measurement or if the coefficient of variation of the ten scans was greater than $25 \%$. The median value at each wavelength was used in subsequent calculations, to reduce any remaining effects of outliers (Cuthill et al., 1999). 


\section{Data analysis}

Color perception by living organisms is based largely on interactions between cones in the retina of the eye (Dowling, 1987; Endler, 1990). Unfortunately, the details of how different classes of cones interact have been adequately worked out for only a very few species (Endler, 1990). We have therefore chosen to use a color space model for analyzing color changes. The color space model has been used in a variety of studies, for example on fish and bees, to examine color perception (e.g. Menzel \& Backhaus, 1989; Neumeyer, 1992). This model is generic in that it does not depend on a comprehensive knowledge of the visual physiology of the animal but only on the composition and properties of the photoreceptors. We calculated relative spectral sensitivities for each cone from nomograms provided by C. Baube (Baube \& Loew, unpubl.), with peak sensitivities at wavelengths of: L (long wavelength), $598 \mathrm{~nm} ; \mathrm{M}$ (medium), $530 \mathrm{~nm} ; \mathrm{S}$ (short), $438 \mathrm{~nm}$; UV (ultraviolet), $362 \mathrm{~nm}$. For each cone we then calculated its absolute stimulation by each color pattern, i.e. reflectance* cone sensitivity summed across wavelengths. We next calculated relative stimulation by dividing absolute stimulation of, say, the L cone, by the sum of absolute stimulation for all four classes of cone (e.g. $\mathrm{L} /[\mathrm{L}+\mathrm{M}+\mathrm{S}+\mathrm{UV}])$. In principle, enhancement of chroma occurs (and differences in hue are perceived) when relative stimulation of a cone class increases or decreases.

A further set of calculations allowed us to begin to investigate how contrast between color patches might influence perception of each as well as overall conspicuousness, again from the perspective of the stickleback. We calculated the Euclidean distance between color space measures for the eye and jaw with and without social cues. The assumption is that the greater the Euclidean difference the greater the contrast likely perceived by the fish. This is similar to the measure that Endler (1991) and Lythgoe \& Partridge (1991) used for calculating contrast: $\mathrm{D}=\left(\operatorname{Sum}\left(\mathrm{St}_{\text {mouth }}[\mathrm{C}]-\mathrm{St}_{\text {eye }}[\mathrm{C}]\right)^{2}\right)^{1 / 2}$, for stimulation, 'St' by the four 'C', cones, L-UV. It is a summary measure of differences, between contexts, in the relative stimulation of each of the cones forming the basis of color perception.

We conducted an additional analysis with respect to the interaction between the $\mathrm{L}$ and $\mathrm{M}$ cones, which are numerically dominant in the stickleback retina, should be especially important in the perception of red and have been emphasized in previous analyses of stickleback jaw and belly coloration (Bakker \& Mundwiler, 1994; Baube et al., 1995). For the L and $\mathrm{M}$ cones, we calculated a measure of differential stimulation $(\mathrm{ConeL}-\mathrm{ConeM}) /(\mathrm{ConeL}+$ ConeM) (Baube et al., 1995). A high 'LM' value should contribute to a perception of higher red chroma and more extreme red hue on the part of the stickleback. To quantify contrast in terms of this measure, we also calculated 'LM contrast', LM for the jaw minus LM for the eye, for each social context. This is somewhat similar to the Euclidean distance calculations but emphasizes differences in the relative stimulation of the two major cone types in the stickleback eye (in human terms, a low or negative value of LM should contribute to perception of green, or potentially blue if the $\mathrm{S}$ cone is still more strongly stimulated).

As no data are available for the threespine stickleback, we do not incorporate effects of intra-ocular absorbance in our analyses. In another Gasterosteid, Spinachia spinachia, lens absorbance is minimal for the wavelengths considered in this study (Thorpe \& Truscott, 1993). In the absence of data on natural lighting conditions for our study population, we also omit calculations based on ambient light and beam attenuation (Endler, 1990) from our analyses.

Unless otherwise indicated, all results are given as two-tailed paired $t$-tests, with sequential bonferroni corrections of tests of the same hypothesis with different cones. 


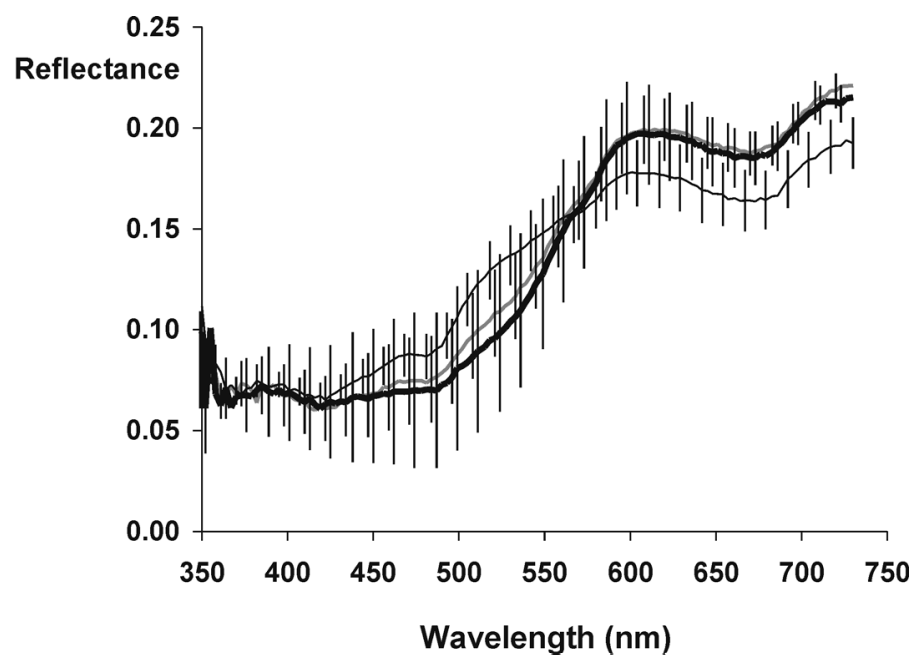

Fig. 1. Mean proportion reflectance from jaws of anadromous male sticklebacks. Grey line: with female conspecific stimulus; thick black line: with male conspecific stimulus; thin black line: with no social stimuli (average of no male and no female data, to simplify figure). Error bars: SE's.

\section{Results}

Jaw and eye reflectance patterns change with social context

Inspection of the reflectance data (Fig. 1,2) reveals that eye and jaw color patterns are strikingly different and this difference increases in the presence of conspecifics. When a mature male or female is present, the peak reflectance of the eye shifts further toward short 'blue' wavelengths from slightly longer 'green' wavelengths and reflectance at long wavelengths is generally decreased. When a mature male or female is present, jaw reflectance at long wavelengths is increased and the transition to low reflectance at short wavelengths becomes more abrupt, increasing the saturation of male red coloration. Patterns in the UV (350-400 nm) are mainly extensions of patterns in the human-visible spectrum, for both the jaw and the eye, and reflectances in the UV are low. At the shortest wavelengths in our analyses, just above $350 \mathrm{~nm}$, there is a narrow spike for both color patches. Possibly this is meaningful, but reflectance data at these very short wavelengths were somewhat noisy and marginally reliable, owing to low illumination.

The patterns present in the reflectance data are echoed in analyses using the generalized color space model of stickleback visual functioning. For the 


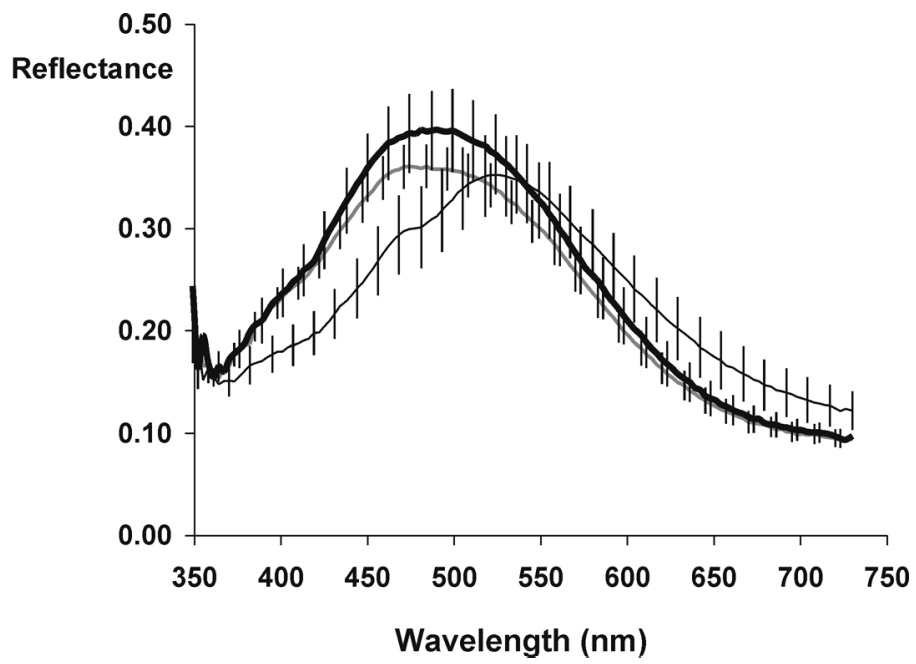

Fig. 2. Mean proportion reflectance from eyes of anadromous male sticklebacks. Grey line: with female conspecific stimulus; thick black line: with male conspecific stimulus; thin black line: with no social stimuli (average of no male and no female data, to simplify figure). Error bars: SE's.

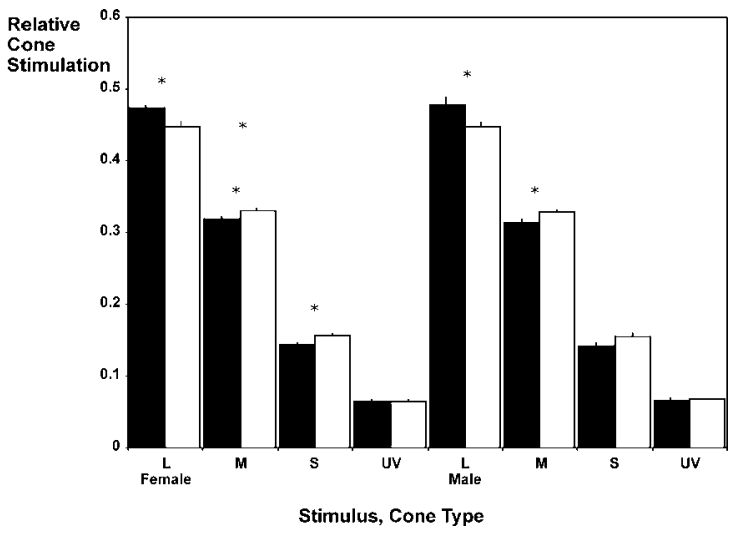

Fig. 3. Relative calculated stimulation of sticklebacks cones by light reflected from jaws of males in presence (dark) or absence (light) of a male or female stimulus, as indicated. Star indicates statistical significance at $p<0.05$ after Bonferroni correction for each stimulus pair, male stimulus $v s$ no male or female stimulus $v s$ no female; female $v s$ male stimulus, no male $v s$ no female, were never significantly different. $N=10$ in all cases. Error bars: SE's.

jaw (Fig. 3), the L cone was stimulated more with conspecifics present, and the $\mathrm{M}$ cone was stimulated significantly less. The $\mathrm{S}$ cone was stimulated less, 


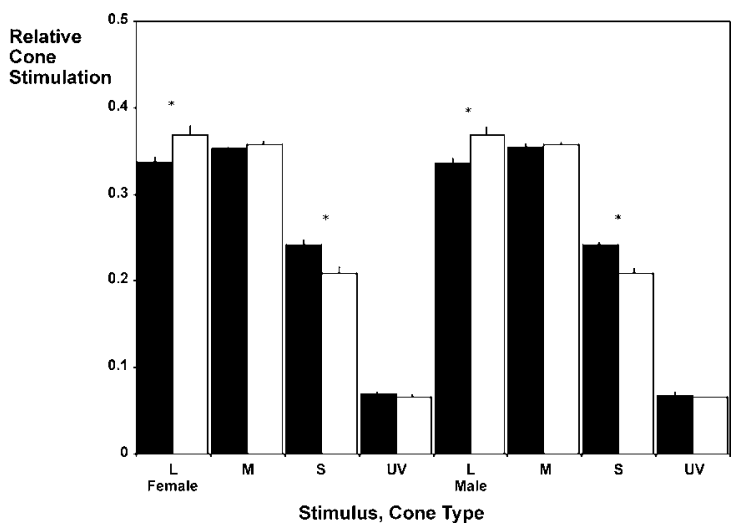

Fig. 4. Relative calculated stimulation of sticklebacks cones by light reflected from eyes of males in presence (dark) or absence (light) of a male or female stimulus, as indicated. Star indicates statistical significance at $p<0.05$ after Bonferroni correction for each stimulus pair, male stimulus $v s$ no male or female stimulus $v s$ no female; female $v s$ male stimulus, no male $v s$ no female, were never significantly different. $N=10$ in all cases. Error bars: SE's.

but this result was significant only with a female stimulus. The UV cone did not exhibit a statistically significant pattern. Whether the conspecific was male or female did not affect cone stimulation significantly in any case (paired $t$-tests, $p>0.1$ in every case, without Bonferroni correction; $N=$ 10).

For the eye (Fig. 4), stimulation of the $\mathrm{S}$ cone increased significantly in the presence of a male or female conspecific. Stimulation of the $\mathrm{L}$ cone decreased significantly whereas there were no significant changes in the stimulation of the $\mathrm{M}$ or UV cones. Whether the conspecific was male or female did not affect cone stimulation in any analysis (paired $t$-tests, $p>$ 0.15 in every case, without Bonferroni correction; $N=10$ ).

\section{Estimates of perceived contrast increase with social stimuli}

The Euclidean distance measures of contrast increased significantly in the presence of either a male ( $p=0.00008$, paired $t$-test, $N=10$ ) or female ( $p=0.0005$, paired $t$-test, $N=10$ ) conspecific, relative to no social stimulus (Fig. 5). Thus, contrast between jaw and eye coloration increased strongly and consistently with social stimulation.

LM values, which are essentially chroma measures focusing on the two main cone classes, reflected the patterns observed for the jaw and eye independently as well as in terms of the contrast between them. LM values 


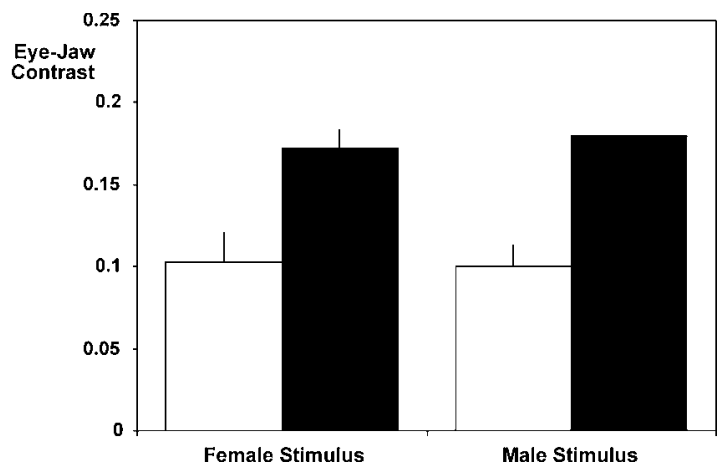

Fig. 5. Mean Euclidean distance measures of eye-jaw contrast in different social contexts. Light: no social stimulus. Dark: male or female social stimulus, as indicated. Error bars: SE's.

decreased significantly with the presence of a conspecific for the eye (paired $t$-tests, $p=0.02,0.001$ for female and male stimuli, $N=10$; data not presented) but increased significantly for the jaw (paired $t$-tests, $p=0.002$ for both female and male stimuli, respectively, $N=10$ ). The increase was similar for male and female conspecifics and LM values did not differ with conspecific gender (paired $t$-tests, $p=0.15,0.35$ for eye and jaw, respectively, $N=10$ ). As might be expected from these results there was a significant increase in LM contrast in the presence of a conspecific (paired $t$-test, $p=0.001,0.00008$ for female, male stimuli, respectively, $N=10$ ). As in previous analyses, the gender of the conspecific did not have a significant effect (paired $t$-test, $p=0.27, N=10$ ). In addition, there was a significant negative correlation between eye and jaw LM values with a female stimulus (Fig. 6: $r=-0.69, p<0.05, N=10$ ) though the relationship was weaker and non-significant with a male stimulus $(r=-0.20, p>0.5, N=10)$. The correlation results indicate, at least for the female stimulus, that individuals with strong positive LM values for the jaw have strong negative LM values for their eyes. Thus, eye and jaw LM may not only change significantly in response to social stimulation, their coloration may be coordinated to enhance contrast for individuals with the most intensely red jaws and blue eyes.

\section{Condition and coloration}

We did not observe a positive relationship between condition and indices of the red coloration of the jaw. With a positive relationship, stimulation of the 


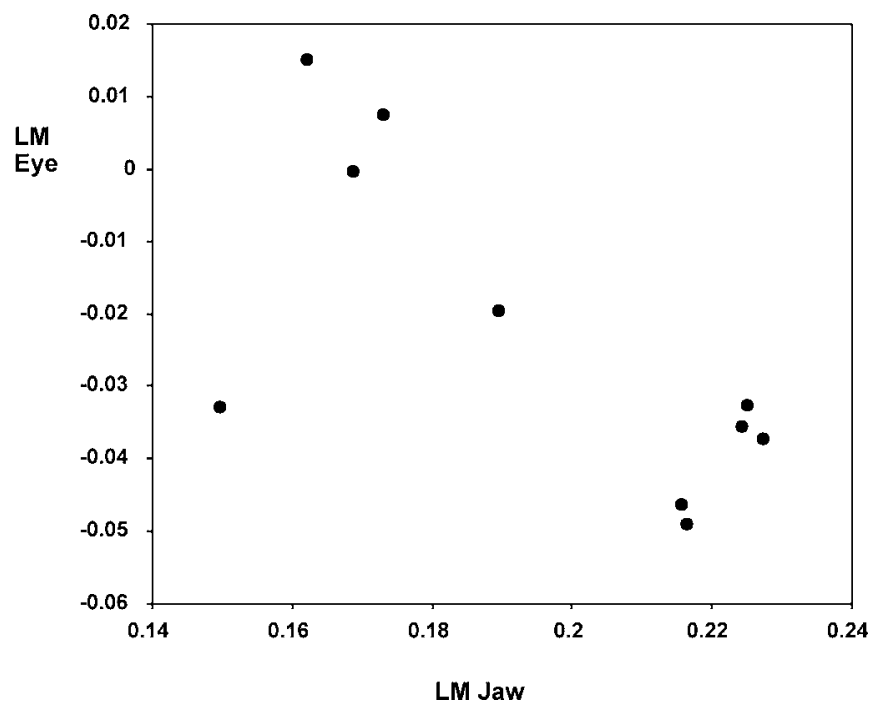

Fig. 6. Correlation between eye and jaw 'LM' (relative stimulation of long wavelength, or red, cone - stimulation of medium wavelength, or green, cone) of males with a female.

long wavelength cone would be expected to increase while stimulation of the medium and short wavelength cones would be expected to decrease. In fact, just the opposite pattern was observed (Table 1) with significant negative correlations between stimulation of the long wavelength cone and condition, with no female present, and a significant positive correlation between stimulation of the short wavelength cone and condition in this context. The same patterns were apparent with no male present, but were significant only for the short wavelength cone. Surprisingly, no significant results were obtained in the presence of a conspecific male or female.

Given Candolin's (1999b) reports of better fed, high condition males showing greater reduction of red coloration in response to predators, we also asked if the color signal of high condition males may be generally more dynamic and change more with social context. If so, there should be correlations between condition and the magnitude of color change when a conspecific is present versus absent (e.g. correlation between relatively long wavelength cone stimulation with a female present minus stimulation without a female, and condition). Just such a correlation was observed, and was significant for the long wavelength cone for the female-no female comparison and almost significant for the short wavelength cone $(p<0.06$ : Table 1). Thus, high condition males reduced their long wavelength reflectance and 
TABLE 1. Correlations between condition and relative cone stimulation

\begin{tabular}{lcccccr}
\hline Cone & \multicolumn{6}{c}{ Stimulus } \\
\cline { 2 - 7 } & $\begin{array}{l}\text { Female } \\
\text { Stimulus }\end{array}$ & $\begin{array}{c}\text { No } \\
\text { Female }\end{array}$ & $\begin{array}{c}\text { Male } \\
\text { Stimulus }\end{array}$ & $\begin{array}{c}\text { No } \\
\text { Male }\end{array}$ & $\begin{array}{c}\text { Female-No } \\
\text { Female }\end{array}$ & $\begin{array}{c}\text { Male-No } \\
\text { Male }\end{array}$ \\
\hline L (aka Red) & -0.142 & $-0.833^{*}$ & -0.3 & -0.71 & $0.801^{*}$ & 0.468 \\
M (aka Green) & -0.447 & 0.023 & -0.196 & -0.141 & -0.633 & -0.104 \\
S (aka Blue) & 0.348 & $0.904^{*}$ & 0.212 & $0.824^{*}$ & -0.762 & -0.562 \\
UV & 0.33 & 0.462 & 0.577 & 0.469 & -0.402 & 0.228 \\
\hline
\end{tabular}

$N=9$ in all cases.

${ }^{*} p<0.05$; bonferroni correction applied to each column.

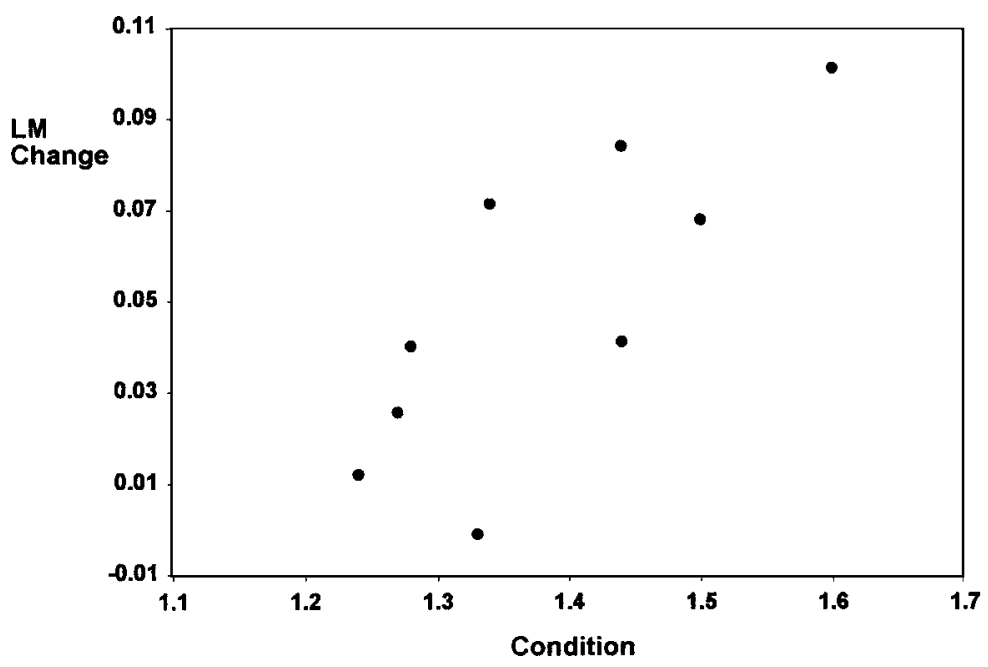

Fig. 7. Correlation between condition and difference, in the presence versus the absence of a female, in jaw 'LM' (relative stimulation of long wavelength, or red, cone - stimulation of medium wavelength, or green, cone).

tended to enhance reflectance at short wavelengths, in the absence of a female, whereas low condition males tended to alter their coloration less. As might be expected from these results, change in LM between social contexts was also correlated with condition (Fig. 7: $r=0.78, N=9, p<0.02$ ). Results for male stimulus/no stimulus were consistently in the same direction but slightly weaker in magnitude and not significant (Table 1; for LM correlation $r=0.43, N=9, p>0.2)$. 


\section{Discussion}

Our study provides the first documentation, not reliant upon human-based indices, of social effects on stickleback coloration and the first such data from unrestrained males. We find that social stimuli affect both eye and jaw coloration. In the case of the eye, the reflectance pattern shifts to a shorter peak wavelength and relatively stronger stimulation of the $S$ ('blue') cone when a conspecific is present. This result also demonstrates that stickleback eye coloration is highly labile and may be difficult to measure reliably from restrained fish. Our analyses of jaw reflectance confirm previous reports of an increased intensity of male red coloration in the presence of conspecifics (e.g. McLennan \& McPhail, 1990). Our observation that males elevate their eye and jaw color saturation in response to both male and female conspecifics is consistent with other evidence that stickleback coloration functions in both intersexual and intrasexual interactions (McLennan \& McPhail, 1990; Rowland, 1994; Baube, 1997).

Most studies of sticklebacks have emphasized red coloration or have considered jaw and eye coloration in isolation, but our analyses suggest that interactions between red ventro-lateral areas and blue eye color may be important in the perception of each and in maximizing overall conspicuousness. We find that as the eye becomes more blue and the throat more red with social stimulation, the contrast between the two is also elevated. Because our measure of contrast is based on the visual physiology of the fish, the result is probably real in terms of stickleback perception. Further, our results suggest that the most intensely red males also possess the most saturated blue eyes (though the result was significant only for the female stimulus), consistent with the hypothesis that contrast between the two is selected for when conspicuousness is favored as well as with some earlier data (McLennan \& McPhail, 1989a, b). Other color pattern elements may also play a substantial role in enhancing the apparent intensity of red coloration and overall conspicuousness. In some red-throated stream populations, non-red lateral areas may develop an intense blue sheen and in some cases become black (Bell, 1982; McKinnon, 1995; Von Hippel, 1999). Thus, a more comprehensive evaluation of additional elements of stickleback color patterns may be important in future work, as with guppies (Endler, 1991; Brooks \& Endler, 2001).

Ganglion cells, one of the color coding units of the fish visual system, may be a mechanism by which enhanced contrast between color pattern elements 
stimulates the stickleback eye and brain. Some ganglion cells in goldfish respond preferentially to red coloration in the center of a circle and green coloration in the surrounding area. Other ganglion cells respond strongly to blue and green in the center of the field and red in the surround (Daw, 1968). The shift in coloration that we see would maximally stimulate a ganglion cell that has its center around the eye and its broad surround near the throat; or vice versa. While our results are only qualitative with respect to ganglion cell function, they suggest a degree of sensory exploitation of stickleback visual processing by the male signal. Detailed analysis of the stickleback visual system would clearly go a long way to fully testing this idea.

Our results do not suggest a special or unique role for UV reflectance from the stickleback eye and jaw, unlike some other fishes (Kodric-Brown \& Johnson, 2002; Macías Garcia \& de Perera, 2002) and birds (Bennet et al., 1996; Sheldon et al., 1999). Certainly UV reflectance is present, especially for the eye, and it could influence potential mates. However, UV-reflectance is at a lower level than for human-visible wavelengths, and there are no obvious changes in reflectance in the UV, simply continuation of patterns present in the visible spectrum. Also, the relative stimulation of the UV cone did not change with social context in our analyses, in contrast to results for those cones that are most sensitive at human-visible wavelengths. It is also noteworthy that $\mathrm{L}, \mathrm{M}$ and $\mathrm{S}$ cones exhibit substantial sensitivity in the $\mathrm{UV}$, but relative sensitivities of these cones are more similar in the UV than for visible wavelengths (Baube \& Loew, unpubl.). Sensitivity in the UV accounts in part for the high relative stimulation obtained for the $\mathrm{L}$ cone even with light reflected from the blue eye of the stickleback. UV reflectance from other areas of the stickleback body, and at still shorter wavelengths, remains to be investigated.

The relationships between condition, health and red coloration in sticklebacks have been much studied and discussed, but remain contentious (e.g. Milinski \& Bakker, 1990; Rowland, 1994). In this, the first study of these relationships using reflectance data and a color vision model based on stickleback visual physiology, we did not find a positive relationship between condition and red intensity. Despite our small sample size, however, we found support for a more dynamic signal in high condition males, much as Candolin (1999b) documented in response to predators. Thus, high condition males reduce their red intensity more in the absence of a female conspecific. 
It remains to be seen whether this result is obtained because high condition males are of intrinsically superior quality and therefore investing differently in present and future reproduction - or whether the low condition males have simply been through more breeding cycles and color both intensifies and loses flexibility throughout the breeding season for possibly non-adaptive reasons (Candolin, 1999b). It is also interesting to speculate whether females can detect the ability of males to alter their coloration, and whether such flexibility is attractive.

Our results suggest that meaningful reflectance data can be collected from unrestrained threespine sticklebacks. Thus, it is possible to obtain measures of coloration that allow evaluation of the influence of social context and other factors associated with transient color changes. Moreover, reflectance data can be analyzed in terms of their perception by conspecifics or other species such as predators, even for light not visible to humans. In future studies, male coloration might be analyzed during more complete interactions with females and perhaps other males, in which case it may not be necessary to use parental males as experimental subjects. They were used here in order to insure that males returned to the nest for color-measurement when standardized stimuli were presented. In addition, it may be possible for similar methods to be applied to different fish species or other vertebrates. Protocols could be developed that allow individuals to be coaxed into particular positions in which standardized color measurements can be taken; or some training of experimental subjects could be conducted.

These analyses omit several details, such as ocular absorbance and wavelength-specific absorption of light by water, which might influence relative cone stimulation (Endler, 1990, 1991; Baube et al., 1995). Addition of such details to future studies would be valuable. We would be surprised, however, if there were substantial changes to our most central finding, that social stimuli affect stickleback coloration so as to cause greater differential cone stimulation and higher 'chroma' for each color patch as well as higher contrast between the jaw and eye. On the other hand, a more thorough understanding of the details of stickleback color vision, and in particular how color perception varies among populations and environments (McDonald \& Hawryshyn, 1995; Boughman, 2001), would greatly enhance the interpretation of our reflectance results. It would also be worthwhile to investigate the effect of angles of illumination and observation on reflectance from the eye, and whether aquarium glass could have more subtle effects on reflectance data than simply absorbing light at some wavelengths. 


\section{References}

Andersson, M. (1994). Sexual selection. - Princeton University Press, Princeton.

Bagnara, J.T. \& Hadley, M.E. (1973). Chromatophores and color change: the comparative physiology of animal pigmentation. - Prentice-Hall, Englewood Cliffs, NJ.

Bakker, T.C.M. \& Mundwiler, B. (1994). Female mate choice and male red coloration in a natural three-spined stickleback (Gasterosteus aculeatus) population. - Behav. Ecol. 5, p. 74-80.

Baube, C.L. (1997). Manipulations of signalling environment affect male competitive success in three-spined sticklebacks. - Anim. Behav. 53, p. 819-833.

— —, Rowland, W.J. \& Fowler, J.B. (1995). The mechanisms of colour-based mate choice in female threespine sticklebacks: hue, contrast and configurational cues. - Behaviour 132, p. 979-996.

Bell, M.A. (1982). Melanism in a high elevation stream population of Gasterosteusaculeatus. - Copeia 1982, p. 829-835.

Bennett, A.T.D., Cuthill, I.C. \& Noriss, K.J. (1994). Sexual selection and the mismeasure of color. - Am. Nat. 144, p. 848-860.

_ _, _ —, Partridge, J.C. \& Maier, E.J. (1996). Ultraviolet vision and mate choice in zebra finches. - Nature 380, p. 433-435.

Bolger, T. \& Connolly, P.L. (1989). The selection of suitable indices for the measurement and analysis of fish condition. - J. Fish Biol. 34, p. 171-182.

Boughman, J.W. (2001). Divergent sexual selection enhances reproductive isolation in sticklebacks. - Nature 411, p. 944-948.

Brooks, R. \& Endler, J.A. (2001). Direct and indirect sexual selection and quantitative genetics of male traits in guppies (Poecilia reticulata). - Evolution 55, p. 1002-1015.

Candolin, U. (1999a). Male-male competition facilitates female choice in sticklebacks. Proc. R. Soc. Lond. B 266, p. 785-789.

- - (1999b). The relationship between signal quality and physical condition: is sexual signalling honest in the three-spined stickleback? - Anim. Behav. 58, p. 1261-1267.

- - (2000). Increased signalling effort when survival prospects decrease: male-male competition ensures honesty. - Anim. Behav. 60, p. 417-422.

Cuthill, I.C., Bennett, A.T.D., Partridge, J.C. \& Maier, E.J. (1999). Plumage reflectance and the objective assessment of avian sexual dichromatism. - Am. Nat. 160, p. 183-200.

Daw, N.W. (1968). Colour-coded ganglion cells in the goldfish retina. Extension of their receptive fields by means of new stimuli. - J. Physiol. Lond. 197, p. 567-592.

Dowling, J.E. (1987). The retina, an approachable part of the brain. — Harvard University Press, Cambridge, MA.

Endler, J.A. (1990). On the measurement and classification of colour in studies of animal colour patterns. - Biol. J. Linn. Soc. 41, p. 315-352.

- - (1991). Variation in the appearance of guppy color patterns to guppies and their predators under different visual conditions. - Vis. Res. 31, p. 587-608.

Frischknecht, M. (1993). The breeding colouration of male three-spined sticklebacks (Gasterosteus aculeatus) as an indicator of energy investment in vigour. - Evol. Ecol. 7, p. 439-450.

Fuji, R. \& Novales, R.R. (1969). Cellular aspects of the control of physiological color change in fishes. - Amer. Zool. 9, p. 453-463. 
Hagen, D.W. (1967). Isolating mechanisms in threespine sticklebacks (Gasterosteus). J. Fish. Res. Bd. Can. 24, p. 1637-1692.

Kodric-Brown, A. \& Johnson, S.C. (2002). Ultraviolet reflectance patterns of male guppies enhance their attractiveness to females. - Anim. Behav. 63, p. 391-396.

Kraak, S.B.M., Bakker, T.C.M. \& Mundwiler, B. (1999). Correlates of the duration of the egg collecting phase in the three-spined stickleback. - J. Fish Biol. 54, p. 1038-1049.

Losey, G.S., Cronin, T.W., Goldsmith, T.H., Hyde, D., Marshall, N.J. \& McFarland, W.N. (1999). The UV visual world of fishes: a review. - J. Fish Biol. 54, p. 921-943.

Lythgoe, J.N. \& Partridge, J.C. (1991). The modelling of optimal visual pigments of dichromatic teleosts in green coastal waters. - Vision Res. 31, p. 361-371.

Macías Garcia, C. \& de Perera, T.B. (2002). Ultraviolet-based female preferences in a viviparous fish. - Behav. Ecol. Sociobiol. 52, p. 1-6.

McDonald, C.G. \& Hawryshyn, C.W. (1995). Intraspecific variation of spectral sensitivity in threespine stickleback (Gasterosteus aculeatus) from different photic regimes. J. Comp. Phys. A 176, p. 255-260.

McKinnon, J.S. (1995). Video mate preferences of female three-spined sticklebacks from populations with divergent male coloration. - Anim. Behav. 50, p. 1645-1655.

- - (1996). Male parental behavior and red coloration in the threespine stickleback, Gasterosteus aculeatus. — J. Fish Biol. 49, p. 1030-1033.

McLennan, D.A. \& McPhail, J.D. (1989a). Experimental investigations of the evolutionary significance of sexually dimorphic nuptial colouration in Gasterosteus aculeatus (L.): temporal changes in the structure of the male mosaic signal. — Can. J. Zool. 67, p. 17671777 .

- — \& - (1989b). Experimental investigations of the evolutionary significance of sexually dimorphic nuptial colouration in Gasterosteus aculeatus (L.): the relationship between male colour and male behaviour. - Can. J. Zool. 68, p. 482-492.

- — \& — - (1990). Experimental investigations of the evolutionary significance of sexually dimorphic nuptial colouration in Gasterosteus aculeatus (L.): the relationship between male colour and female behaviour. - Can. J. Zool. 68, p. 482-492.

McPhail, J.D. (1969). Predation and the evolution of a stickleback (Gasterosteus). — J. Fish. Res. Bd. Canada 26, p. 3183-3208.

Menzel, R. \& Backhaus, W. (1989). Color vision in honey bees: phenomena and physiological mechanisms. - In: Facets of vision (D.G. Stavenga \& R.C. Hardie, eds). SpringerVerlag, Berlin, p. 281-297.

Milinski, M. \& Bakker, T.C.M. (1990). Female sticklebacks use male coloration in mate choice and hence avoid parasitized males. - Nature 344, p. 330-333.

Neumeyer, C. (1992). Tetrachromatic color vision in goldfish: evidence from color mixture experiments. - J. Comp. Physiol. A 171, p. 639-649.

Rowland, W.J. (1994). Proximate determinants of stickleback behaviour: an evolutionary perspective. - In: Evolutionary biology of the threespine stickleback (M.A. Bell \& S.A. Foster, eds). Oxford University Press, Oxford, p. 297-344.

Sheldon, B.C., Andersson, S., Griffith, S.C., Ornborg, J. \& Sendecka, J. (1999). Ultraviolet colour variation influences blue tit sex ratios. - Nature 402, p. 874-877.

Smith, E.J., Partridge, J.C., Parsons, K.N., White, E.M., Cuthill, I.C., Bennett, A.T.D. \& Church, S.C. (2002). Ultraviolet vision and mate choice in the guppy (Poecilia reticulata). - Behav. Ecol. 13, p. 11-19. 
Thorpe, A., Douglas, R.H. \& Truscott, R.J.W. (1993). Spectral transmission and short-wave absorbing pigments in the fish lens - I. Phylogenetic distribution and identity. Vision Res. 33, p. 289-300.

Tinbergen, N. (1951). The study of instinct. - Oxford University Press, Oxford.

Von Hippel, F.A. (1999). Black male bellies and red female throats: color changes with breeding status in a threespine stickleback. - Env. Biol. Fishes 55, p. 237-244.

Wootton, R.J. (1976). The biology of the sticklebacks. — Academic Press, London. 